\title{
Correction: an embryo-specific expressing TGF- $\beta$ family protein, growth-differentiation factor 3 (GDF3), augments progression of B16 melanoma
}

Nobuyuki Ehira ${ }^{1,2}$, Hiroyuki Oshiumi ${ }^{*}$, Misako Matsumoto ${ }^{1}$, Takeshi Kondo $^{2}$, Masahiro Asaka ${ }^{2}$ and Tsukasa Seya ${ }^{1}$

\section{Correction}

In Figure two of our article [1], panel A inadvertently contains a duplication of panel B. This error was made during the arrangement of the figure panels. The corrected, revised Figure two (Figure 1 here) is shown below. The error does not affect any conclusions drawn
(A)

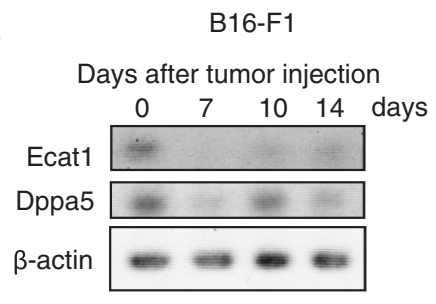

(C)

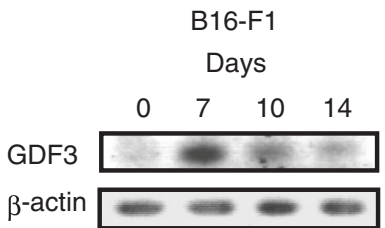

$(\mathrm{E})$

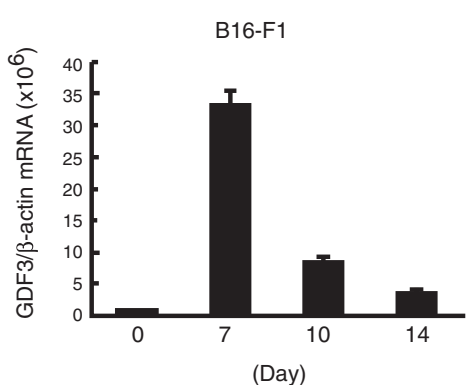

(B)

B16-F10

Dasy after tumor injection

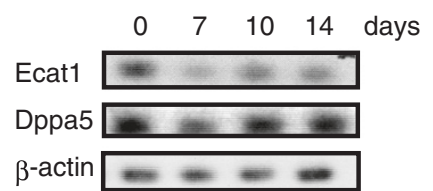

(D)

B16-F10

Days

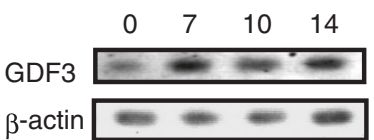

$(\mathrm{F})$

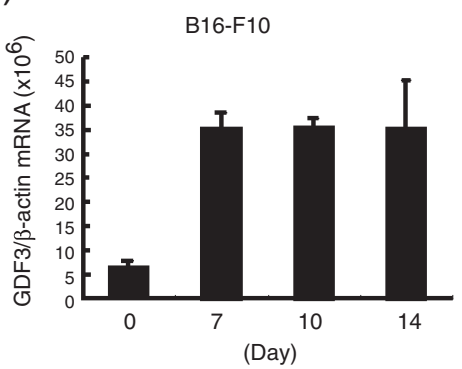

Figure 1 Expression kinetics of Ecat1, Dppa5, and GDF3 during tumorigenesis. B16-F1 and B16-F10 cells were injected subcutaneously into C57BL/6 mice. Tumors were excised on the indicated day. Total RNA was extracted from the tumor and RT-PCR (A-D) or RT-qPCR (E, F) was performed to detect Ecat1, Dppa5, and GDF3. (A, B) RT-PCR analyses revealed that mRNA of Eca1 and Dppa5 decreased during tumorigenesis. (C, E) In B16-F1 cells, GDF3 peaked at day 7 after tumor injection and then gradually decreased. (D, F). In contrast, GDF3 expression in B16-F10 cells increased 7 days after tumor injection and maintained a high level until 14 days after injection.

* Correspondence: oshiumi@med.hokudai.ac.jp

'Department of Microbiology and Immunology, Graduate School of

Medicine, Hokkaido University, Kita-15, Nishi-7, Kita-ku, Sapporo 060-8638, Japan 
in the article. We regret any inconvenience this has caused.

\section{Author details}

'Department of Microbiology and Immunology, Graduate School of Medicine, Hokkaido University, Kita-15, Nishi-7, Kita-ku, Sapporo 060-8638, Japan. ${ }^{2}$ Department of Gastroenterology, Graduate School of Medicine, Hokkaido University, Kita-15, Nishi-7, Kita-ku, Sapporo 060-8638, Japan.

Received: 18 February 2014 Accepted: 18 February 2014

Published: 21 February 2014

\section{Reference}

1. Ehira N, Oshiumi H, Matsumoto M, Kondo T, Asaka M, Seya T: An embryospecific expressing TGF- $\beta$ family protein, growth-differentiation factor 3 (GDF3), augments progression of B16 melanoma. J Exp Clin Cancer Res 2010, 29:135.

doi:10.1186/1756-9966-33-22

Cite this article as: Ehira et al.: Correction: an embryo-specific expressing TGF- $\beta$ family protein, growth-differentiation factor 3 (GDF3), augments progression of B16 melanoma. Journal of Experimental \& Clinical Cancer Research 2014 33:22. 